\title{
Updated checklist of Iranian Braconidae (Hymenoptera: Ichneumonoidea) with twenty-three new records
}

\author{
${ }^{1}$ Najmeh Samin, ${ }^{2}$ Juana María Coronado-Blanco, ${ }^{3}$ Maximilian Fischer, ${ }^{4}$ Kees van Achterberg, \\ ${ }^{5}$ Hamid SAKENIN \& 6 ELENA Davidian
}

\author{
${ }^{1}$ Young Researchers and Elite Club, Science and Research Branch, Islamic Azad University, Tehran, Iran; \\ email: n_samin63@yahoo.com \\ ${ }^{2}$ Facultad de Ingeniería y Ciencias, Universidad Autónoma de Tamaulipas, Mexico; \\ email: jmcoronado@docentes.uat.edu.mx
}

${ }^{3}$ Hofrat i.R. Univ.-Dozent, Naturhistorisches Museum, 2. Zoologische Abteilung, A-1010 Wien, Burgring 7 , Austria; email: maximilian.fischer@chello.at

${ }^{4}$ Department of Entomology, National Natuurhistorisch Museum, Postbus 9517, 2300 RA Leiden, The Netherlands;email: c.vanachterberg@xs4all.nl

${ }^{5}$ Department of Plant Protection, Qaemshahr Branch, Islamic Azad University, Mazandaran, Iran; email: hchelave@yahoo.com

${ }^{6}$ Russian Institute of Plant Protection (VIZR), Podbelsky shosse, 3, St. Petersburg-Pushkin, 196608, Russia; email: gdavidian@yandex.ru

Samin N., Coronado-Blanco J. M., Fischer M., van Achterberg K., Sakenin H. \& Davidian E.: Updated checklist of Iranian Braconidae (Hymenoptera: Ichneumonoidea) with twenty-three new records.

Abstract: This faunistic paper deals with species diversity of nine subfamilies of Braconidae (Hymenoptera) of Iran. A total of 23 species within 18 genera were collected and identified as new records for the fauna of Iran: Agathidinae (two species), Alysiinae (two species), Aphidiinae (one species), Braconinae (one species), Cenocoelionidae (two species), Euphorinae (four species), Microgastrinae (seven species), Opiinae (three species) and Sigalphinae (one species). Additionally, updated checklist of Iranian Braconidae included 861 species is represented in this checklist.

Keywords: Species diversity, catalogue, fauna, new country records, parasitoids, Iran

\section{Introduction}

Braconid wasps (Hymenoptera: Braconidae) are one of the most diverse and powerful parasitoids which have efficient role in biological control of various agricultural and forest pests (MaTthews 1974, SHAw 1995, QuicKe et al. 1999). This family comprises 21,221 described species and with estimation of 40,000 to 50,000 species worldwide (YU et al. 2016). The members of the family Braconidae are primary parasitoids on other insects, especially the larval stages of Coleoptera, Diptera, and Lepidoptera, but also some hemimetabolous insects like aphids, Heteroptera or Embiidina (SHARKEY 1993, WHARTON 1993). 
The fauna of Iranian Braconidae was studied rather well under several contributions and also increasing the faunistic surveys in recent years (e.g., GHAHARI et al. 2011, 2012a, b, RAstegar et al. 2012, SAKENIN et al. 2012, FARAHANI et al. 2013, 2014, AMERI et al. 2014a, b; SAMIN et al. 2014, 2015, 2016; GHAHARI \& GADAllah 2015; GHAHARI \& Van Achterberg 2016, Ghahari \& Beyarslan 2017, Sakenin Chelav et al. 2018). Most of the subfamilies were catalogued by GADALlaH \& GHAHARI (2013a, b, 2015, 2016, 2017), Gadallah et al. (2015a, b, 2016a, b), and Beyarslan et al. (2017). FARAHANI et al. (2016) listed 780 species in 141 genera, and after that Yu et al. (2016) listed 804 species for the fauna of Iran. The aim of this paper is introducing of 23 new country records, and updating of the Iranian Braconidae checklist.

\section{Material and methods}

The specimens from different regions of Iran were collected by Malaise traps, sweeping and light trapping, were put in ethanol $75 \%$, pined or mounted on small labels, according to taxonomic methods and were determined. Morphological terminology followed Tobias (1986), VAn ACHTERberg (1993), Belokobylskij (1998) and Chen et al. (2004). Classification and nomenclature are derived from Yu et al. (2016).

\section{Results}

In this paper, totally 23 species of Braconidae within nine subbamilies, Agathidinae (two species, two genera), Alysiinae (two species, two genera), Aphidiinae (one species), Braconinae (one species), Cenocoelionidae (two species, two genera), Euphorinae (four species, four genera), Microgastrinae (seven species, four genera), Opiinae (three species, one genus) and Sigalphinae (one species) were collected and identified from different regions of Iran

Subfamily Agathidinae Haliday, 1833

Genus Agathis Latreille, 1805

Agathis pedias Nixon, 1986

Material examined: Luristan province, Azna, $33^{\circ} 28^{\prime} \mathrm{N} 49^{\circ} 25^{\prime} \mathrm{E}, 1$,, $6 . v i i .2016$.

Lepton nigrum (Nees, 1812)

Genus Lepton Turton, 1822

Material examined: Fars province, Fasa, $28^{\circ} 58^{\prime} \mathrm{N} 53^{\circ} 46^{\prime} \mathrm{E}, 1$ ㅇ, 11.v.2011.

Subfamily Alysiinae Leach, 1815

Genus Chorebus Haliday, 1833

Chorebus (Stiphrocera) dagda (Nixon, 1943)

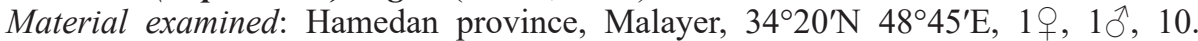
viii.2013.

Genus Coloneura Förster, 1862

Coloneura arestor (Nixon, 1954)

Material examined: Zanjan province, Abhar, $36^{\circ} 16^{\prime} \mathrm{N} 49^{\circ} 03^{\prime} \mathrm{E}, 2$, 9 , 22.vi.2015. 
Aphidius auctus Haliday, 1833

Subfamily Aphidiinae Haliday, 1833

Genus Aphidius Nees, 1819

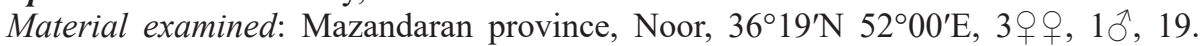
vii.2010.

Subfamily Braconinae Nees von Esenbeck, 1811

Genus Bracon Fabricius, 1804

Bracon (Glabrobracon) oscillator (Nees von Esenbeck 1812)

Material examined: Qazvin province, Takestan, $36^{\circ} 00^{\prime} \mathrm{N} 49^{\circ} 33^{\prime} \mathrm{E}, 1 \stackrel{\circ}{ }, 2 \bigcirc^{\Uparrow}, 21$. vi.2015.

Cenocoelius analis (Nees, 1834)

Subfamily Cenocoelionidae Szépligeti, 1901

Genus Cenocoelius Westwood, 1840

Material examined: Esfahan province, Shahr-e-Reza, $32^{\circ} 01^{\prime} \mathrm{N} 51^{\circ} 87^{\prime} \mathrm{E}, 1^{\circ}$, 29.v.2011.

Genus Lestricus Reinhard, 1865

Lestricus secalis (Linnaeus, 1758)

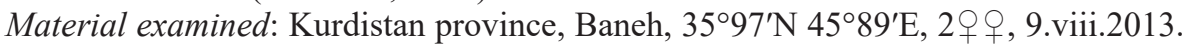

Subfamily Euphorinae Foerster, 1863

Genus Marshiella Shaw, 1985

Marshiella plumicornis (Ruthe, 1856)

Material examined: Chahar-Mahal and Bakhtiari province, Farsan, $19,32^{\circ} 15^{\prime} \mathrm{N}$ $50^{\circ} 34^{\prime} \mathrm{E}, 15-16$ vii.2013.

Meteorus lionotus Thomson, 1895

Material examined: Fars province, Eghlid, $30^{\circ} 53^{\prime} \mathrm{N} 52^{\circ} 41^{\prime} \mathrm{E}, 2$, 9 ㅇ, 5.v.2009; Esfahan

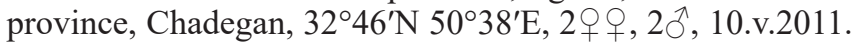

Genus Peristenus Foerster, 1863

Peristenus facialis (Thomson, 1892)

Material examined: Khorasan-e Razavi province, Ferdos, $34^{\circ} 10^{\prime} \mathrm{N} 57^{\circ} 40^{\prime} \mathrm{E}, 2$, 9 , 19.vi.2013.

Genus Streblocera Westwood, 1833

Streblocera (Streblocera) fulviceps Westwood, 1833

Material examined: Khuzestan province, Andimeshk, $32^{\circ} 35^{\prime} \mathrm{N} 48^{\circ} 20^{\prime} \mathrm{E}, 1 \mathrm{O}^{\wedge}$, 7.v.2015.

Subfamily Microgastrinae Förster, 1862

Tribe Apantilini Viereck, 1918

Genus Apanteles Förster, 1862

Apanteles aragatzi Tobias, 1976

Material examined: Kurdistan province, Qorveh, $35^{\circ} 15^{\prime} \mathrm{N} 47^{\circ} 40^{\prime} \mathrm{E}, 1$, , 9.viii.2013.

Apanteles atreus Nixon, 1973

Material examined: Khuzestan province, Shushtar, $32^{\circ} 00^{\prime} \mathrm{N} 48^{\circ} 50^{\prime} \mathrm{E}, 1$, , 14.v.2014.

Apanteles impurus (Nees, 1834)

Material examined: East Azarbaijan province, Shabestar, $38^{\circ} 12^{\prime} \mathrm{N} 45^{\circ} 44^{\prime} \mathrm{E}, 2 \circ q$, 11.viii.2016, ex Scrobipalpa sp. (Lepidoptera: Gelechiidae). 
Genus Cotesia Cameron, 1891

Cotesia gonopterygis (Marshall, 1897)

Material examined: Kurdistan province, Qorveh, $35^{\circ} 15^{\prime} \mathrm{N} 47^{\circ} 40^{\prime} \mathrm{E}, 1 \stackrel{+}{ }, 1 \delta^{\wedge}, 9$. viii.2013.

Genus Microplitis Förster, 1863

Microplitis albipennis Abdinbekova, 1969

Material examined: Khuzestan province, Andimeshk, $32^{\circ} 35^{\prime} \mathrm{N} 48^{\circ} 20^{\prime} \mathrm{E}, 2 \stackrel{\circ}{\circ}, 2{ }^{\top}$, 7.v.2015

Microplitis mandibularis (Thomson, 1895)

Material examined: Fars province, Eghlid, 30 $53^{\prime} \mathrm{N} 52^{\circ} 41^{\prime} \mathrm{E}, 2$ 우, ${ }^{\circ}$.v.2009.

Genus Protapanteles Ashmead, 1898

Protapanteles acasta (Nixon, 1973)

Material examined: Khorasan-e Shomali province, Shirvan, $37^{\circ} 32^{\prime} \mathrm{N} 57^{\circ} 54^{\prime} \mathrm{E}, 1^{\circ}$, 1ふ̄, 27.vi.2015.

Subfamily Opiinae Blanchard, 1845

Genus Opius Wesmael, 1835

Opius (Allophlebus) fuscipennis Wesmael, 1835

Material examined: Fars province, Fasa, $28^{\circ} 58^{\prime} \mathrm{N} 53^{\circ} 46^{\prime} \mathrm{E}, 2$, $ᄋ$, 11.v.2011.

Opius (Cryptonastes) gracilis Fischer, 1957

Material examined: Khuzestan province, Shushtar, $32^{\circ} 00^{\prime} \mathrm{N} 48^{\circ} 50^{\prime} \mathrm{E}, 3+9+1{ }^{\top}$, 14.v.2014, ex Amauromyza (Cephalomyza) gyrans (Fallen, 1823) (Diptera: Agromyzidae).

Opius (Utetes) rotundiventris (Thomson, 1895)

Material examined: Markazi province, Khomein, $33^{\circ} 40^{\prime} \mathrm{N} 50^{\circ} 00^{\prime} \mathrm{E}, 1$ 우 , 25.vii.2015.

Subfamily Sigalphinae Haliday, 1833

Genus Sigalphus Latreille, 1802

Sigalphus caudatus Nees, 1816

Material examined: Hamedan province, Razan, $35^{\circ} 25^{\prime} \mathrm{N} 49^{\circ} 00^{\prime} \mathrm{E}, 1^{\wedge}{ }^{\prime}, 3.1 x .2012$.

\section{Discussion}

This investigation with 23 new records proves that the fauna of Iranian Braconidae is diverse and on the other hand, unknown yet. Regarding to the new records of this faunistic research, the total species number of Iranian Braconidae reaches to 861 (YU et al. 2016: 804 species; BEYARSLAN et al. 2017: five new records; GADALLAH \& GHAHARI 2017: five new records; GHAHARI \& VAN ACHTERBERG 2016: one new record; GHAHARI \& BEYARSLAN 2017: two new records; SAKENIN et al. 2018: 21 new records; and this study: 23 new records). Among the different subfamilies of Iranian Braconidae, Braconinae with 122 recorded species and subspecies is more diverse than all others, followed by Microgastrinae, Opiinae and Alysiinae with 111, 104 and 94 species/subspecies, respectively (Fig. 1).

Iran is a large country $\left(1,623,779 \mathrm{~km}^{2}\right)$ and is characterized by a high climatic heterogeneity (HaKimZADEH KHOEI et al. 2011). It is bordered in the north by the Caucasus Mountains, Middle Asian natural regions and the Caspian Sea, in the west by the Anatolian and Mesopotamian regions, in the east by the eastern part of the Iranian plateau and the Baluch-Sindian region, and finally in the south by the Persian Gulf and Oman Sea, which are connected by the latter to the Indian Ocean (ZEHZAD et al. 2002). 


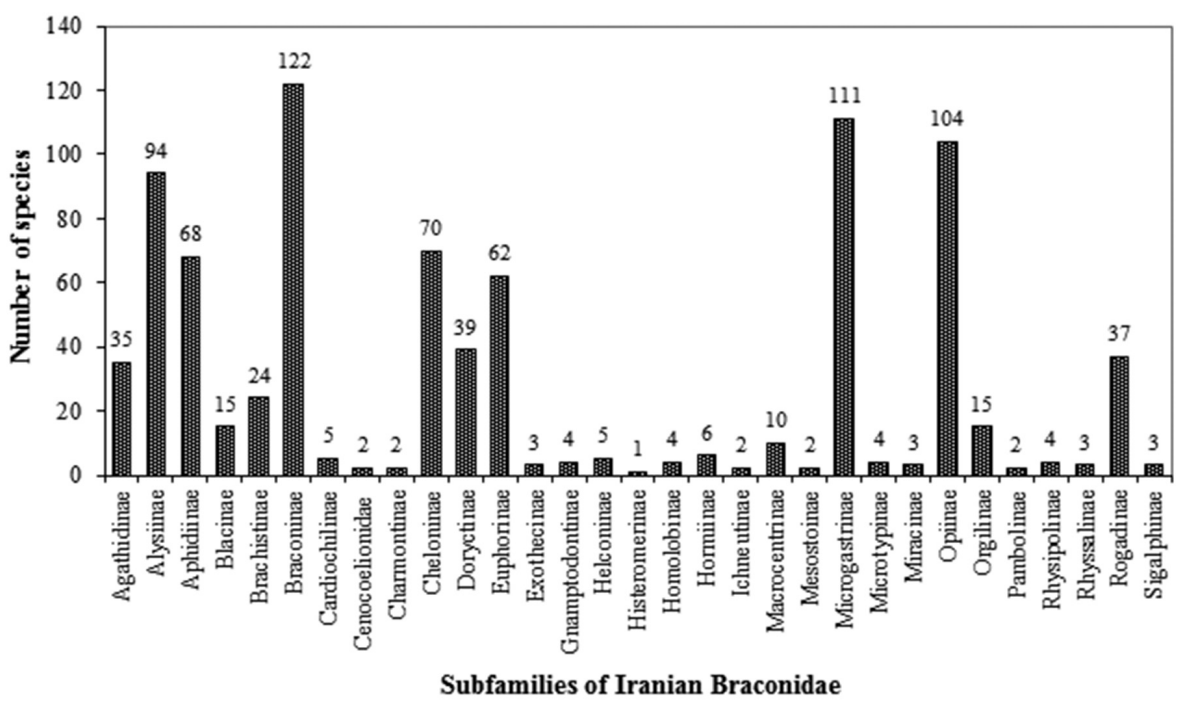

Fig. 1: Species diversity of Iranian Braconidae

The biogeographic location of Iran in the Palaearctic is unique, placed at the crossroads of the Oriental and Afrotropical ecozones, Central Asia and Caucasus. Therefore, its fauna is expected to be mainly South Palaearctic, but with representatives of Central Asian, Caucasus, Oriental and Afrotropical elements (Strumia \& FallahZadeH 2015, FALlahZADEH \& JAPOSHVILI 2017). Continuing of faunistic surveys in different regions especially in the unsampled areas (e.g., northeastern and central parts of Iran) will result to finding new data (new country records, new species, new host records). Regarding to high diversity of braconids' hosts in Iran (Coleoptera, Lepidoptera and Diptera), we expect several unknown species in different ecosystems. Braconid wasps are powerful parasitoids of agricultural and forest pests which can decrease the population densities of different pests especially Lepidoptera. Conservation of these natural enemies will be resulted to higher efficiency of these beneficial insects.

\section{Acknowledgements}

The authors are grateful to A. Beyarslan (Trakya University, Turkey), J. Papp (Hungarian Natural History Museum) for identification of some materials, H. Ghahari (Iran) for reviewing the manuscript, Dicky S. Yu (Canada) and S.R. Shaw (USA) for providing of some literature. The research was supported by Islamic Azad University (Young Researchers and Elites Club, Science and Research Branch), and Universidad Autónoma de Tamaulipas. 


\section{References}

VAN ACHTERBERG, C. 1993: Illustrated key to the subfamilies of the Braconidae (Hymenoptera: Ichneumonoidea). - Zoologische Verhandelingen, 283, 189 pp.

Ameri, A., Talebi, A. A., Rakhshani, E., Beyarslan, A. \& Kamali, K. 2014a: A survey of Euphorinae (Hymenoptera: Braconidae) of southern Iran, with description of a new species. - Zootaxa 3900: 415-428.

Ameri, A., Talebi, A.A., Rakhshani, E., Beyarslan, A. \& Kamali, K. 2014b: Taxonomic study of the subfamily Doryctinae (Hymenoptera: Braconidae) in Hormozgan province, southern Iran. - Zoology and Ecology 24: $40-54$.

BelokobylskiJ, S.A. 1998: 1. Rhyssalinae, 2. Doryctinae, 3. Histeromerinae, 4. Exothecinae, 7. Gnamptodontinae, 9. Alysiinae (Alysiini), 10. Helconinae, 11. Cenocoeliinae, 12. Brachistinae, 14. Meteorideinae, 16. Xiphozelinae, 17. Homolobinae, 18. Charmontinae, 19. Orgilinae, 20. Ecnomiinae, 21. Sigalphinae, 23. Ichneutinae, 25. Cardiochilinae, 27. Dirrhopinae, 28. Miracinae, 29. Adeliinae. In: Ler, P.A. ,Key to the insects of Russian Far East. Vol. 4. Neuropteroidea, Mecoptera, Hymenoptera. Part 3. Dal'nauka, Vladivostok, 706 pp.

Beyarslan, A., Gadallah, N. S. \& Ghahari, H. 2017: An annotated catalogue of the Iranian Microtypinae and Rogadinae (Hymenoptera: Braconidae). - Zootaxa 4291: 099-116.

Chen, X., He, J. \& MA, Y. 2004. Fauna Sinica. Insecta Vol. 37. Hymenoptera. Braconidae (II). Science Press, Beijing, China, $581 \mathrm{pp}$.

Fallahzadeh, M. \& Japoshvili, G. 2017: An updated checklist of Iranian Encyrtidae (Hymenoptera, Chalcidoidea). - Zootaxa 4344(1): 1-46.

Farahani, S., TAlebi, A.A. \& Rakhshani, E. 2013. A contribution to the tribe Chelonini Foerster (Hymenoptera: Braconidae: Cheloninae) of northern Iran, with first records for eight species and an updated checklist of Iranian species. - Zoosystematics and Evolution 89(2): 227-238.

Farahani, S., Talebi, A.A. \& Rakhshani, E. 2014. Wasps of the subfamily Doryctinae (Hymenoptera: Braconidae) in Iran. - Zoology in the Middle East 60(1): 65-81.

Farahani, S., TAlebi, A.A. \& Rakhshani, E. 2016: Iranian Braconidae (Insecta: Hymenoptera: Ichneumonoidea): diversity, distribution and host association. - Journal of Insect Biodiversity and Systematics 2: 1-92.

Gadallah, N. S. \& Ghahari, H. 2013a: An annotated catalogue of the Iranian Agathidinae and Brachistinae (Hymenoptera: Braconidae). - Linzer biologische Beiträge 45/2: 1873-1901.

Gadallah, N.S. \& GHAhari, H. 2013b: An annotated catalogue of the Iranian Cheloninae (Hymenoptera: Braconidae). - Linzer biologische Beiträge 45/2: 1921-1943.

Gadallah, N. S. \& Ghahari, H. 2015: An annotated catalogue of the Iranian Braconinae (Hymenoptera: Braconidae). - Entomofauna 36: 121-176.

Gadallah, N. S., Ghahari, H., Fischer, M. \& Peris-Felipo, F.J. 2015a: An annotated catalogue of the Iranian Alysiinae (Hymenoptera: Braconidae). - Zootaxa 3974: 001-028.

Gadallah, N. S., Ghahari, H. \& Peris-Felipo, F.J. 2015b: Catalogue of the Iranian Microgastrinae (Hymenoptera: Braconidae). - Zootaxa 4043: 1-69.

Gadallah, N. S., Ghahari, H., Peris-Felipo, F. J. \& Fischer, M. 2016a: Updated checklist of Iranian Opiinae (Hymenoptera: Braconidae). - Zootaxa 4066: 1-40.

Gadallah, N. S., Ghahari, H. \& van Achterberg, C. 2016b: An annotated catalogue of the Iranian Euphorinae, Gnamptodontinae, Helconinae, Hormiinae and Rhysipolinae (Hymenoptera: Braconidae). Zootaxa 4072: 001-038.

Gadallah, N. S. \& Ghahari, H. 2016: An updated checklist of the Iranian Miracinae, Pambolinae and Sigalphinae (Hymenoptera: Braconidae). - Orsis 30: 51-61.

Gadallah, N. S. \& Ghahari, H. 2017: An annotated catalogue of the Iranian Doryctinae and Exothecinae (Hymenoptera: Braconidae). - Transactions of the American Entomological Society 143: 669-691.

Ghahari, H. \& Gadallah N. S. 2015: A faunistic study on the Braconidae (Hymenoptera) from some regions of Semnan, Iran. - Entomofauna 36: 177-184.

Ghahari, H., Fischer, M. \& PAPP, J. 2011: A study on the braconid wasps (Hymenoptera: Braconidae) from Isfahan province, Iran. - Entomofauna 32: 261-270.

Ghahari, H., Fischer, M. \& Tobias, V. 2012a: A contribution to the knowledge of braconids (Hymenoptera: Braconidae) from Lorestan province, Iran. - Entomofauna 33: 65-72.

Ghahari, H., Fischer, M. \& Tobias, V. 2012b: A study on the Braconidae (Hymenoptera: Ichneumonoidea) from Guilan province, Iran. - Entomofauna 33: 317-324. 
Ghahari, H. \& van Achterberg, C. 2016: A contribution to the study of subfamilies Microgastrinae and Opiinae (Hymenoptera: Braconidae) from the Arasbaran Biosphere Reserve and vicinity, Northwestern Iran. - Natura Somogyiensis 28: 23-32.

Ghahari, H. \& Beyarslan, A. 2017: A faunistic study on Braconidae (Hymenoptera: Ichneumonoidea) from Iran. - Natura Somogyiensis 30: 39-46.

Hakimzadeh Khoei, M., Kaya, M. \& Altindag, A. 2011: New records of Rotifers from Iran with biogeographic considerations. - Turkish Journal of Zoology 35: 395-402.

Matthews, R.W. 1974. Biology of Braconidae. - Annual Review of Entomology 19: 15-32.

Quicke, D. L. J., Basibuyk, H. H., Fitton, M. G. \& Rasnitsyn, A. P. 1999: Morphological, palaeontological and molecular aspects of ichneumonoid phylogeny (Hymenoptera, Insecta). - Zoologica Scripta 28: 175202.

Rastegar, J., Sakenin, H., Khodaparast, S. \& Havaskary, M. 2012: On a collection of Braconidae (Hymenoptera) from East Azarbaijan and vicinity, Iran. - Calodema 226: 1-4.

Sakenin, H., Naderian, H., Samin, N., Rastegar, J., Tabari, M. \& Papp, J. 2012: On a collection of Braconidae (Hymenoptera) from northern Iran. - Linzer Biologische Beiträge 22: 1319-1330.

Sakenin Chelav, H., Coronado-Blanco, J. M., Samin, N. \& Fischer, M. 2018. New records of Braconidae (Hymenoptera) from Iran. - Far Eastern Entomologist 362: 13-16.

Samin, N., Ghahari, H., Gadallah, N.S. \& Davidian, E. 2014: A study on the Braconidae (Hymenoptera: Ichneumonoidea) from West Azarbaijan province, Northwestern Iran. - Linzer Biologische Beiträge 46: 1447.1678.

SAMIN, N., Fischer, M. \& GHAHARI, H. 2015: A contribution to the study on the fauna of Braconidae (Hymenoptera, Ichneumonoidea) from the province of Semnan, Iran. - Arquivos Entomoloxicos 13: 429-433.

Samin, N., van Achterberg, C. \& Erdogan, O.C. 2016: A faunistic study on some subfamilies of Braconidae (Hymenoptera: Ichneumonoidea) from Iran. - Arquivos Entomolóxicos 15: 153-161.

Sharkey, M. J. 1993: Family Braconidae, pp. 362-395 In: Goulet, H. \& Huber, J. T. (eds), Hymenoptera of the world: An identification guide to families. - Agriculture Canada, vii+668 pp.

Shaw, M. R. \& Huddleston, T. 1991: Classification and biology of braconid wasps (Hymenoptera: Braconidae). - Handbooks of the Identification of British Insects 7: 1-126.

Strumia, F. \& Fallahzadeh, M. 2015: New records and three new species of Chrysididae (Hymenoptera, Chrysidoidea) from Iran. - Journal of Insect Biodiversity 3(15): 1-32.

Tobias, V.I. 1986: Order Hymenoptera. Family Braconidae. In: Medvedev, G. S. (ed.), 'Opredelitel Nasekomych Evrospeiskoi Tsasti SSSR 3, Peredpontdatokrylye 4. Opr. Faune SSSR.’ 145: 1-501. [Keys to the insects of the European part of USSR. Hymenoptera]. [English translation. Lebanon, USA].

Wharton, R. A. 1993. Bionomics of the Braconidae. - Annual Review of Entomology 38: 121-143.

Yu, D. S., van Achterberg, C. \& Horstmann, K. 2016: Taxapad 2016, Ichneumonoidea 2016, Database on flash-drive, Ottawa, Ontario, Canada.

Zehzad, B., Kiabi, B. \& Madjnoonian, H. 2002: The natural areas and landscape of Iran: an overview. Zoology in the Middle East 26(1): 7-10. 


\section{Appendix 1. Subfamilies, genera and species of Braconidae that occure in Iran (new records are marked with an asterisk (*)).}

\section{Subfamily Agathidinae}

1) Agathis anglica Marshall, 1885

2) A. assimilis Kokujev, 1895

3) A. breviseta Nees, 1812

4) A. fulmeki Fischer, 1957

5) A. fuscipennis (Zetterstedt, 1838)

6) A. glaucoptera Nees, 1834

7) A. lugubris (Förster, 1863)

8) A. malvacearum Latreille, 1805

9)A. melpomene Nixon, 1986

10) A. montana Shestakov, 1932

11) A. nigra Nees, 1812

12) A. pedias Nixon, $1986 *$

13) A. pumila (Ratzeburg, 1844)

14) A. rostrata Tobias, 1963

15) A. rufipalpis Nees, 1812

16) A. semiaciculata Ivanov, 1899

17) A. syngenesiae Nees, 1812

18) A. tibialis Nees, 1812

19) A. umbellatarum Nees, 1812

20) A. varipes Thomson, 1895

21) Camptothlipsis armeniaca (Telenga, 1955)

22) Coccygidium transcaspicum (Kokujev, 1902)

23) Cremnops desertor (Linnaeus, 1758)

24) C. richteri Hedwig, 1957

25) Disophrys caesa (Klug, 1835)

26) D. dissors Kokujev, 1903

27) D. inculcatrix (Kriechbaumer, 1898)

28) Earinus elator (Fabricius, 1804)

29) Lepton nigrum (Nees, 1812)*

30) Lytopylus persicus Farahani \& Talebi, 2014

31) L. rufipes (Nees, 1812)

32) Therophilus clausthalianus (Ratzeburg, 1844)

33) T. dimidiator (Nees, 1834)

34) T. linguarius (Nees, 1812)

35) T. tumidulus (Nees, 1812)

\section{Subfamily Alysiinae}

36) Adelurola amplidens (Fischer, 1966)

37) Alloea contracta (Haliday, 1833)

38) Alysia rufidens Nees, 1834

39) Angelovia elipsocubitalis Zaykov, 1980

40) Aphaereta difficilis Nixon, 1939

41) A. minuta (Nees, 1811)

42) Aristelix persica Peris-Felipo, 2015

43) Aspilota alfalfae Fischer, Lashkari Bod, Rakhshani \& Talebi, 2011

44) A. delicata Fischer, 1973

45) A. isfahanensis Peris-Felipo, 2016

46) Carinthilota vechti van Achterberg, 1988

47) Chorebus affinis (Nees, 1812)

48) Ch. aphantus (Marshall, 1896)

49) Ch. ares (Nixon, 1944)

50) Ch. asphodeli Griffiths, 1968
51) Ch. axillaris Fischer, Lashkari Bod, Rakhshani \& Talebi, 2011

52) Ch. baeticus Griffiths, 1967

53) Ch. bathyzonus (Marshall, 1895)

54) Ch. caesariatus Griffiths, 1967

55) Ch. calthae Griffiths, 1967

56) Ch. compressiiventris (Telenga, 1935)

57) Ch. cubocephalus (Telenga, 1935)

58) Ch.dagda (Nixon, 1943)*

59) Ch. diremtus (Nees, 1834)

60) Ch. femoratus (Tobias, 1962)

61) Ch. flavipes (Goureau, 1851)

62) Ch. fuscipennis (Nixon, 1937)

63) Ch. gedanensis (Ratzeburg, 1852)

64) Ch. gracilipes (Thomson, 1895)

65) Ch. groschkei Griffiths, 1967

66) Ch. heringianus Griffiths, 1967

67) Ch. iridis Griffiths, 1968

68) Ch. lar (Morley, 1924)

69) Ch. leptogaster (Haliday, 1839)

70) Ch. longiarticulis Fischer, Lashkari Bod, Rakhshani \& Talebi, 2011

71) Ch. merellus (Nixon, 1937)

72) Ch. misellus (Marshall, 1895)

73) Ch. mucronatus (Telenga, 1935)

74) Ch. nigridiremptus Fischer, Lashkari Bod, Rakhshani \& Talebi, 2011

75) Ch. nigriscaposus (Nixon, 1949)

76) Ch. nixoni Burghele, 1959

77) Ch. ornatus (Telenga, 1935)

78) Ch. parvungula (Thomson, 1895)

79) Ch. posticus (Haliday, 1839)

80) Ch. properesam Fischer, Lashkari Bod, Rakhshani \& Talebi, 2011

81) Ch. pseudomisellus Griffiths, 1968

82) Ch. scabiosae Griffiths, 1967

83) Ch. solstitialis (Stelfox, 1951)

84) Ch. spenceri Griffiths, 1964

85) Ch. stilifer Griffiths, 1968

86) Ch. tamsi (Nixon, 1944)

87) Ch. tumidus (Tobias, 1966)

88) Ch. uliginosus (Haliday, 1839)

89) Ch. venustus (Tobias, 1962)

90) Ch. zarghanensis Fischer, Lashkari Bod, Rakhshani \& Talebi, 2011

91) Coelinidea elegans (Curtis, 1829)

92) C. gracilis (Curtis, 1829)

93) Coloneura arestor (Nixon, 1954)*

94) C. dice (Nixon, 1943)

95) Dacnusa abdita (Haliday, 1839)

96) D. alpestris Griffiths, 1967

97) D. aterrima Thomson, 1895

98) D. clematidis Griffiths, 1967

99) D. confinis Ruthe, 1859 
100) D. evadne Nixon, 1937

101) D. gentianae Griffiths, 1967

102) D. heringi Griffiths, 1967

103) D. hospita (Förster, 1863)

104) D. monticola (Förster, 1863)

105) D. pubescens (Curtis, 1826)

106) D. sasakawai Takada, 1977

107) D. sibirica Telenga, 1935

108) Dinotrema amoenidens (Fischer, 1973)

109) D. concinnum (Haliday, 1838)

110) D. concolor (Nees, 1812)

111) D. contracticorne (Fischer, 1974)

112) D. cratocera (Thomson, 1895)

113) D. distractum (Nees, 1834)

114) D. intermissum (Fischer 1974)

115) D. significarium (Fischer, 1973)

116) Exotela umbellina (Nixon 1954)

117) Idiasta picticornis (Ruthe, 1854)

118) Orthostigma beyarslani Fischer, 1995

119) O. laticeps (Thomson, 1895)

120) O. maculipes (Haliday, 1838)

121) Phaenocarpa brevipalpis (Thomson, 1895)

122) Ph. ruficeps (Nees, 1812)

123) Polemochartus liparae (Giraud, 1863)

124) Protodacnusa aridula (Thomson, 1895)

125) Protodacnusa litoralis Griffiths, 1964

126) Pseudopezomachus cursitans (Ferrière, 1930)

127) P. masii Nixon, 1940

128) Synaldis megastigma Fischer, 1967

129) S. ultima Fischer, 1970

\section{Subfamily Aphidiinae}

130) Adialytus ambiguus (Haliday, 1834)

131) A. salicaphis (Fitch, 1855)

132) A. thelaxis (Starý, 1961)

133) A. veronicaecola (Starý, 1978)

134) Aphidius arvensis (Starý, 1960)

135) A. asteris Haliday, 1834

136) A. auctus Haliday, $1833 *$

137) A. cingulatus Ruthe, 1859

138) A. colemani Viereck, 1912

139) A. eadyi Starý, González \& Hall, 1980

140) A. ervi Haliday, 1834

141) A. funebris Mackauer, 1961

142) A. hieraciorum Starý, 1962

143) A. iranicus Rakhshani \& Starý, 2007

144) A. matricariae Haliday, 1834

145) A. persicus Rakhshani \& Starý, 2006

146) A. platensis Brèthes, 1913

147) A. popovi Starý, 1978

148) A. rhopalosiphi de Stefani-Perez, 1902

149) A. rosae Haliday, 1833

150) A. salicis Haliday, 1834

151) A. setiger (Mackauer, 1961)

152) A. smithi Sharma \& Subba Rao, 1959

153) A. stigmaticus Rakhshani \& Tomanovic, 2011

154) A. transcaspicus Telenga, 1958
155) A. urticae Haliday, 1834

156) A. uzbekistanicus Luzhetzki, 1960

157) Areopraon lepelleyi (Waterston, 1926)

158) Betuloxys hortorum (Starý, 1960)

159) Binodoxys acalephae (Marshall, 1896)

160) B. angelicae (Haliday, 1833)

161) B. brevicornis (Haliday, 1833)

162) B. heraclei (Haliday, 1833)

163) Diaeretiella rapae (McIntosh, 1855)

164) Ephedrus cerasicola Starý, 1962

165) E. chaitophori Gärdenfors, 1986

166) E. helleni Mackauer, 1968

167) E. laevicollis (Thomson, 1895)

168) E. niger Gautier, Bonnamour \& Gaumont, 1929

169) E. persicae Froggatt, 1904

170) E. plagiator (Nees, 1811)

171) Lipolexis gracilis Förster, 1863

172) Lysiphlebus confusus Tremblay \& Eady, 1978

173) L. desertorum Starý, 1965

174) L. fabarum (Marshall, 1896)

175) L. testaceipes (Cresson, 1880)

176) Monoctonia vesicarii Tremblay, 1991

177) Monoctonus mali van Achterberg, 1989

178) Pauesia antennata (Mukerji, 1950)

179) P. hazratbalensis Bhagat, 1981

180) Praon abjectum (Haliday, 1833)

181) P. barbatum Mackauer, 1967

182) P. exsoletum (Nees, 1811)

183) P. flavinode (Haliday, 1833)

184) P. necans Mackauer, 1959

185) P. orpheusi Kavallieratos, Athanassiou \& Tomanovic, 2003

186) P. rosaecola Starý, 1961

187) P. unitum Mescheloff \& Rosen, 1989

188) P. volucre (Haliday, 1833)

189) P. yomenae Takada, 1968

190) Tanytrichophorus petiolaris Mackauer, 1961

191) Trioxys asiaticus Telenga, 1953

192) T. cirsii (Curtis, 1831)

193) T. complanatus Quilis, 1931

194) T. pallidus (Haliday, 1833)

195) T. pannonicus Starý, 1960

196) T. persicus Davidian, 2005

197) T. tanaceticola Starý, 1971

\section{Subfamily Blacinae}

198) Blacus achterbergi Haeselbarth, 1976

199) B. armatulus Ruthe, 1861

200) B. bovistae Haeselbarth, 1973

201) B. diversicornis (Nees, 1834)

202) B. errans (Nees, 1811)

203) B. exilis (Nees, 1811)

204) B. filicornis Haeselbarth, 1973

205) B. hastatus Haliday, 1835

206) B. humilis (Nees, 1811)

207) B. interstitialis Ruthe, 1861 
208) B. maculipes Wesmael, 1835

209) B. nixoni Haeselbarth, 1973

210) Blacus paganus Haliday, 1835

211) B. ruficornis (Nees, 1811)

212) B. stelfoxi Haeselbarth, 1973

\section{Subfamily Brachistinae}

213) Diospilus capito (Nees, 1834)

214) D. nigricornis (Wesmael, 1835)

215) D. productus Marshall, 1894

216) Eubazus minutus (Ratzeburg, 1848)

217) E. pallipes Nees, 1812

218) E. tibialis (Haliday, 1835)

219) Foersteria longicauda van Achterberg, 1990

220) Schizoprymnus angustatus (Herrich-Schäffer, 1838)

221) S. brevicornis Herrich-Schäffer, 1838

222) Sch. elongatus (Szépligeti, 1898)

223) Sch. excisus (Snoflák, 1953)

224) Sch. nigripes (Thomson, 1892)

225) Sch. obscurus (Nees, 1816)

226) Sch pallidipennis (Herrich-Schäffer, 1838)

227) Sch. parvus (Thomson, 1892)

228) Sch. pullatus (Dahlbom, 1833)

229) Sch. terebralis (Snoflák, 1953)

230) Taphaeus hiator (Thunberg, 1822)

231) T. complanellae (Hartig, 1847)

232) T. floricola (Wesmael, 1835)

233) T. lugubris Snoflák, 1953

234) T. obscurella (Nees, 1816)

235) T. pallipes (Nees, 1816)

236) Triaspis caucasica Abdinbekova, 1969

\section{Subfamily Braconinae}

237) Atanycolus ivanowi (Kokujev, 1898)

238) Baryproctus zarudnianus Telenga, 1936

239) Bracon abbreviator Nees, 1834

240) B. ahngeri Telenga, 1936

241) B. angustiventris Tobias, 1957

242) B. apricus Schmiedeknecht, 1897

243) B. atrator Nees, 1834

244) B. brevicornis (Wesmael, 1838)

245) B. brevitemporis Tobias, 1959

246) B. chivensis Telenga, 1936

247) B. chrysostigma Greese, 1928

248) B. ciscaucasicus Telenga, 1936

249) B. concolorans Marshall, 1900

250) B. crassicornis Thomson, 1892

251) B. delibator Haliday, 1833

252) B. densipilosus Tobias, 1957

253) B. dichromus (Wesmael, 1838)

254) B. didemie Beyarslan, 2002

255) B. dilatus Papp, 1999

256) B. dolichurus Marshall, 1897

257) B. epitriptus Marshall, 1885

258) B. erraticus (Wesmael, 1838)

259) B. erzurumiensis Beyarslan, 2002
260) B. excisus (Tobias, 1957)

261) B. exhilarator Nees, 1834

262) B. femoralis (Brullé, 1832)

263) B. fortipes (Wesmael, 1838)

264) B. fulvipes Nees, 1834

265) B. grandiceps Thomson, 1892

266) B. gusaricus Telenga, 1933

267) B. guttiger (Wesmael, 1838)

268) B. hebetor Say, 1836

269) B. helleni Telenga, 1936

270) B. hemiflavus Szépligeti, 1901

271) B. humidus Tobias, 1976

272) B. illyricus Marshall, 1888

273) B. immutator Nees, 1834

274) B. intercessor Nees, 1834

275) B. iranicus (Fischer, 1972)

276) B. kirgisorum Telenga, 1936

277) B. kozak Telenga, 1936

278) B. larvicida (Wesmael, 1838)

279) B. lefroyi (Dudgeon \& Gough, 1914)

280) B. leptus Marshall, 1897

281) B. lividus Telenga, 1936

282) B. longicollis (Wesmael, 1838)

283) B. luteator Spinola, 1808

284) B. mariae Dalla Torre, 1898

285) B. meyeri Telenga, 1936

286) B. minutator (Fabricius, 1798)

287) B. necator (Fabricius, 1777)

288) B. negativus Tobias, 1957

289) B. nigratus (Wesmael, 1838)

290) B. nigripilosus Tobias, 1957

291) B. nigriventris (Wesmael, 1838)

292) B. obscurator Nees, 1811

293) B. ophtalmicus Telenga, 1933

294) B. oscillator (Nees, 1812)*

295) B. osculator Nees, 1811

296) B. parvulus (Wesmael, 1838)

297) B. pectoralis (Wesmael, 1838)

298) B. persiangulfensis Ameri, Beyarslan \& Talebi, 2013

299) B. picticornis (Wesmael, 1838)

300) B. piger (Wesmael, 1838)

301) B. pineti Thomson, 1892

302) B. planinotus Tobias, 1957

303) B. popovi Telenga, 1936

304) B. punctithorax Tobias, 1959

305) B. punctifer Thomson, 1892

306) B. quadrimaculatus Telenga, 1936

307) B. radialis (Telenga, 1936)

308) B. robustus Hedwig, 1961

309) B. sabulosus Szépligeti, 1896

310) B. scabriusculus Dalla Torre, 1898

311) B. schmidti Kokujev, 1912

312) B. sphaerocephalus Szépligeti, 1901

313) B. stabilis (Wesmael, 1838)

314) B. subrugosus Szépligeti, 1901

315) B. suchorukovi Telenga, 1936 
316) B. tekkensis Telenga, 1936

317) B. telengai (Mulyarskaya, 1955)

318) B. thuringiacus Schmiedeknecht, 1897

319) B. triangularis Nees, 1834

320) B. trucidator Marshall, 1888

321) B. tschitscherini Kokujev, 1904

322) B. urinator (Fabricius, 1798)

323) B. variator Nees, 1811

324) B. variegator Spinola, 1808

325) Coeloides abdominalis (Zetterstedt, 1838)

326) C. bostrichorum Giraud, 1872

327) C. rossicus (Kokujev, 1902)

328) Glyptomorpha discolor (Thunberg, 1822)

329) G. kasparyani Tobias, 1976

330) G. nachitshevanica Tobias, 1976

331) G. pectoralis (Brullé, 1832)

332) Iphiaulax hians Pérez, 1907

333) I. impeditor (Kokujev, 1898)

334) I. impostor (Scopoli, 1763)

335) I. mactator (Klug, 1817)

336) I. mirabilis (Hedwig, 1957)

337) I. perezi (Fahringer, 1926)

338) I. tauricus Shestakov 1927

339) Megalommum pistacivorae van Achterberg \& Mehrnejad, 2011

340) P. castrator (Fabricius, 1798)

341) P. inscriptor (Nees, 1834)

342) P. kirmanensis (Kokujev, 1907)

343) P. nigrirostris (Kokujev, 1907)

344) P. schaeuffelei (Hedwig, 1957)

245) P. tataricus (Kokujev, 1898)

246) Rhadinobracon zarudnyi (Telenga, 1936)

347) Vipio appellator (Nees, 1834)

348) V. humerator (Costa, 1885)

349) V. illusor (Klug, 1817)

350) V. intermedius Szépligeti, 1896

351) V. longicauda (Boheman, 1853)

352) V. mlokossewiczi Kokujev, 1898

353) V. nomioides Shestakov, 1926

354) V. sareptanus Kawall, 1865

355) V. striolatus Telenga, 1936

356) V. tentator (Rossi, 1790)

357) V. terrefactor (Villers, 1789)

358) V. xanthurus (Fahringer, 1926)

\section{Subfamily Cardiochilinae}

359) Cardiochiles fallax Kokujev, 1895

360) C. saltator (Fabricius, 1781)

361) C. tibialis Hedwig, 1957

362) C. triplus Shenefelt, 1973

363) Pseudcardiochilus abnormipes Hedwig, 1957

\section{Subfamily Cenocoelionidae}

364) Cenocoelius analis (Nees, 1834)*

365) Lestricus secalis (Linnaeus, 1758)*

\section{Subfamily Charmontinae}

366) Charmon cruentatus Haliday, 1833

367) Ch. extensor (Linnaeus, 1758)

\section{Subfamily Cheloninae}

368) Adelius erythronotus (Förster, 1851)

369) A. subfasciatus Haliday, 1833

370) Ascogaster annularis (Nees, 1816)

371) A. bicarinata (Herrich-Schäffer, 1838)

372) A. bidentula Wesmael, 1835

373) A. bimaris Tobias, 1986

374) A. caucasica Kokujev, 1895

375) A. dispar Fahringer, 1934

376) A. disparilis Tobias, 1986

377) A. excavata Telenga, 1941

378) A. gonocephala Wesmael, 1835

379) A. grahami Huddleston, 1984

380) A. kasparyani Tobias, 1976

381) A. klugii (Nees, 1816)

382) A. quadridentata Wesmael, 1835

383) A. varipes Wesmael, 1835

384) Chelonus annulatus (Nees, 1816)

385) Ch. annulipes Wesmael, 1835

386) Ch. armeniacus Tobias, 1976

387) Ch. bidens Tobias, 1972

388) Ch. bonellii (Nees, 1816)

389) Ch. breviventris Thomson, 1874

390) Ch. canescens Wesmael, 1835

391) Ch. carbonator Marshall, 1885

392) Ch. cesa Koçak \& Kemal, 2013

393) Ch. dauricus Telenga, 1941

394) Ch. elongatus Szépligeti, 1898

395) Ch. inanitus (Linnaeus, 1767)

396) Ch. iranicus Tobias, 1972

397) Ch. medus Telenga, 1941

398) Ch. microsomus Tobias, 1964

399) Ch. ocellatus Alexeev, 1971

400) Ch. oculator (Fabricius, 1775)

401) Ch. productus Herrich-Schäffer, 1838

402) Ch. scabrator (Fabricius, 1793)

403) Ch. setaceus Papp, 1993

404) Ch. smirnovi Telenga, 1953

405) Ch. szepligetii Dalla Torre, 1898

406) Ch. wesmaelii Curtis, 1837

407) Microchelonus areolatus (Cameron, 1906)

408) M. basalis (Curtis, 1837)

409) M. cisapicalis Tobias, 1989

410) M. contractus (Nees, 1816)

411) M. depressus (Thomson, 1874)

412) M. erythrogaster (Lucas, 1849)

413) M. flavipalpis (Szépligeti, 1896)

414) M. flavoneavulus (Abdinbekova, 1971)

415) M. incisus Tobias, 1986

416) M. iranicus Tobias, 2001

417) M. kermakiae Tobias, 2001

418) M. milkoi Tobias, 2003

419) M. moczari Papp, 2014 
420) M. mongolicus (Telenga, 1941)

421) M. mucronatus (Thomson, 1874)

422) M. pellucens (Nees, 1816)

423) M. subcontractus (Abdinbekova, 1971)

424) M. subpusillus Tobias, 1997

425) M. telengai (Abdinbekova, 1965)

426) Phanerotoma acuminata Szépligeti, 1908

427) Ph. fracta Kokujev, 1903

428) Ph. katkowi Kokujev, 1900

429) Ph. kozlovi Shestakov, 1930

430) Ph. leucobasis Kriechbaumer, 1894

431) Ph. masiana Fahringer, 1934

432) Ph. minuta Kokujev, 1903

433) Ph. parva Kokujev, 1903

434) $P h$. permixtellae Fischer, 1968

435) $\mathrm{Ph}$. rufescens (Latreille, 1809)

436) Ph. syleptae Zettel, 1990

437) Phanerotomella rufa (Marshall, 1898)

\section{Subfamily Doryctinae}

438) Dendrosoter middendorffii (Ratzeburg, 1848)

439) D. protuberans (Nees, 1834)

440) Dendrosotinus titubatus Papp, 1985

441) Doryctes inopinatus Belokobylskij, 1984

442) D. leucogaster (Nees, 1834)

443) D. striatellus (Nees, 1834)

444) D. undulatus (Ratzeburg, 1852)

445) Doryctodes anticus (Wollaston, 1858) (in Gadallah \& Ghahari, 2017 as Ontsira antica)

446) D. imperator (Haliday, 1836)

447) Ecphylus silesiacus (Ratzeburg, 1848)

448) Hecabalodes radialis Tobias, 1962

449) H. xylophagi Fischer, 1962 - (in Gadallah \& Ghahari, 2017)

450) Hecabolus sulcatus Curtis, 1834

451) Heterospilus cephi Rohwer, 1925

452) H. hemipterus (Thomson, 1892)

453) H. leptosoma Fischer, 1960

454) H. tauricus Telenga, 1941

455) Leluthia asiatica (Tobias, 1980)

456) L. paradoxa (Picard, 1938)

457) L. ruguloscolyti (Fischer, 1962)

458) L. transcaucasica (Tobias, 1976)

459) Ontsira ignea (Ratzeburg, 1852)

460) O. longicaudis (Giraud, 1857)

461) Polystenus rugosus Förster, 1863

462) Rhaconotus aciculatus Ruthe, 1854

463) Rh. kerzhneri Belokobylskij, 1985

464) Rh. pictipennis (Reinhard, 1855) - in Gadallah \& Ghahari, 2017

465) Rh. scaber Kokujev, 1900

466) Rh. testaceus (Szépligeti, 1908)

467) Rh. zarudnyi Belokobylskij, 1990

468) Rhoptrocentrus piceus Marshall, 1897

469) Spathius brevicaudis Ratzeburg, 1844

470) S. exarator (Linnaeus, 1758)
471) S. maderi Fahringer, 1930

472) S. polonicus Niezabitowski, 1910

473) S. rubidus (Rossi, 1794)

474) S. umbratus (Fabricius, 1798)

475) Syngaster lepida Brullé, 1846 - (in Gadallah \& Ghahari, 2017)

476) Zombrus flavipennis (Brullé, 1846)

\section{Subfamily Euphorinae}

477) Allurus lituratus (Haliday, 1835)

478) A. muricatus (Haliday, 1833)

479) Centistes ater (Nees, 1834)

480) C. cuspidatus (Haliday, 1833)

481) C. fuscipes (Nees, 1834)

482) Chrysopophthorus hungaricus (Kiss, 1927)

483) Dinocampus coccinellae (Schrank, 1802)

484) Ecclitura primoris Kokujev, 1902

485) Elasmosoma berolinense Ruthe, 1858

486) E. luxembergense Wasmann, 1909

487) Euphorus basalis (Curtis, 1833)

488) E. pallidistigma (Curtis, 1833)

489) Leiophron deficiens (Ruthe, 1856)

490) L, fascipennis (Ruthe, 1856)

491) L. heterocordyli Richards, 1967

492) Marshiella plumicornis (Ruthe, 1856)*

493) Meteorus affinis (Wesmael, 1835)

494) M. alborossicus Lobodenko, 2000

495) M. breviantennatus Tobias, 1986

496) M. breviterebratus Ameri, Talebi \& Beyarslan, 2014

497) M. cinctellus (Spinola, 1808)

498) M. colon (Haliday, 1835)

499) M. consimilis (Nees, 1834)

500) M. ictericus (Nees, 1811)

501) M. jaculator (Haliday, 1835)

502) M. lionotus Thomson, $1895 *$

503) M. obsoletus (Wesmael, 1835) 9

504) M. pendulus (Müller, 1776)

505) M. pulchricornis (Wesmael, 1835)

506) M. rubens (Nees, 1811)

507) M. rufus (DeGeer, 1778)

508) M. versicolor (Wesmael, 1835)

509) M. vexator (Haliday, 1835)

510) Microctonus aethiops (Nees, 1834)

511) M. colesi Drea, 1968

512) M. melanopus Ruthe, 1856

513) M. morimi (Ferrière, 1931)

514) M. stenocari (Haeselbarth, 2008)

515) Myiocephalus boops (Wesmael, 1835)

516) Neoneurus clypeatus (Förster, 1863)

517) Perilitus brevicollis Haliday, 1835

518) P. falciger (Ruthe, 1856)

519) P. foveolatus Reinhard, 1862

520) P. kokujevi Tobias, 1986

521) P. rutilus (Nees, 1811)

522) P. stelleri (Loan, 1972)

523) Peristenus facialis (Thomson, 1892)* 
524) P. grandiceps (Thomson, 1892)

525) P. nitidus (Curtis, 1833)

526) P. pallipes (Curtis, 1833)

527) P. picipes (Curtis, 1833)

528) P. relictus (Ruthe, 1856)

529) P. rubricollis (Thomson, 1892)

530) Streblocera fulviceps Westwood, 1833*

531) Syntretus elegans (Ruthe, 1856)

532) S. idalius (Haliday, 1833)

533) S. ocularis van Achterberg \& Haeselbarth, 2003

534) S. xanthocephalus (Marshall, 1887)

535) Townesilitus bicolor (Wesmael, 1835)

536) Wesmaelia petiolata (Wollaston, 1858)

537) Zele albiditarsus Curtis, 1832

538) Z. chlorophthalmus (Spinola, 1808)

\section{Subfamily Exothecinae}

539) Colastes braconius Haliday, 1833

540) Shawiana laevis (Thomson, 1892)

541) Xenarcha lustrator (Haliday, 1836) - (in Gadallah \& Ghahari, 2017 as Colastes lustrator)

\section{Subfamily Gnamptodontinae}

542) Gnamptodon breviradialis (Fischer, 1959)

543) $G$. decoris (Förster, 1863)

544) G. georginae (van Achterberg, 1983)

$545 G$. pumilio (Nees, 1834)

\section{Subfamily Helconinae}

546) Aspicolpus carinator (Nees, 1812)

547) A. sibiricus (Fahringer, 1934)

548) Helcon claviventris Wesmael, 1835

549) H. heinrichi Hedqvist, 1967

550) Helconidea dentator (Fabricius, 1804)

\section{Subfamily Histeromerinae}

551) Histeromerus mystacinus Wesmael, 1838

\section{Subfamily Homolobinae}

552) Homolobus annulicornis (Nees, 1834)

553) H. infumator (Lyle, 1914)

554) H. ophioninus (Vachal, 1907)

555) H. truncator (Say, 1829)

\section{Subfamily Hormiinae}

556) Hormisca pseudomitis (Hedwig, 1957)

557) H. tatianae Telenga, 1941

558) Hormius moniliatus (Nees, 1811)

$559 H$. radialis Telenga, 1941

560) H. sculpturatus Tobias, 1967

561) H. similis Szépligeti, 1896

\section{Subfamily Ichneutinae}

562) Ichneutes reunitor Nees, 1816

563) Proterops nigripennis Wesmael, 1835
Subfamily Macrocentrinae

564) Macrocentrus bicolor Curtis, 1833

565) M. blandus Eady \& Clark, 1964

566) M. cingulum Brischke, 1882

567) M. collaris (Spinola, 1808)

568) M. equalis Lyle, 1914

569) M. flavus Vollenhoven, 1878

570) $M$. infirmus (Nees, 1834)

571) $M$. marginator (Nees, 1811)

572) M. resinellae (Linnaeus, 1758)

573) M. thoracicus (Nees, 1811)

\section{Subfamily Mesostoinae}

574) Pseudobiosteres imperfectus Hedwig, 1961

575) Pseudohormius flavobasalis (Hedwig, 1957)

\section{Subfamily Microgastrinae}

576) Apanteles aethiopicus Wilkinson, 1931

577) A. agilla Nixon, 1972

578) A. appellator Telenga, 1949

579) A. aragatzi Tobias, $1976^{*}$

580) A. atreus Nixon, 1973*

581) A. biroicus Papp, 1973

582) A. britannicus Wilkinson, 1941 - (in Ghahari \& van Achterberg, 2016)

583) A. brunnistigma Abdinbekova, 1969

584) A. candidatus (Haliday, 1834)

585) A. carpatus (Say, 1836)

586) A. circumscriptus (Nees, 1834)

587) A. corvinus Reinhard, 1880

588) A. cytherea Nixon, 1972

589) A. decorus (Haliday, 1834)

590) A. dorsalis (Spinola, 1808)

591) A. emarginatus (Nees, 1834)

592) A. exiguus (Haliday, 1834)

593) A. galleriae Wilkinson, 1932

594) A. halidayi Marshall, 1872

595) A. hemara Nixon, 1965

596) A. impurus (Nees, 1834)*

597) A. ingenuoides Papp, 1971

598) A. iranicus Telenga, 1955

599) A. isus Nixon, 1965

600) A. lacteicolor Viereck, 1911

601) A. lacteus (Nees, 1834)

602) A. laspeyresiella Papp, 1972

$603)$ A. longipalpis Reinhard, 1880

604) A. metacarpalis (Thomson, 1895)

605) A. myeloenta Wilkinson, 1937

606) A. nagyi Papp, 1975

607) A. naso Marshall, 1885

608) A. obscurus (Nees, 1834)

609) A. pilosus Telenga, 1955

610) A. schillei Niezabitowski, 1910

611) A. scutellaris Muesebeck, 1921

612) A. seriphia Nixon, 1972

613) A. sicarius Marshall, 1885

614) A. sodalis (Haliday, 1834) 
615) A. subcamilla Tobias, 1976

616) A. suevus Reinhard, 1880

617) A. tedellae Nixon, 1961

618) A. tiro (Reinhard, 1880)

619) A. viminetorum (Wesmael, 1837)

620) Cotesia abjecta (Marshall, 1885)

621) C. ancilla (Nixon, 1974)

622) C. callimone (Nixon, 1974)

623) C. chilonis (Munakata, 1912)

624) C. cuprea (Lyle, 1925)

625) C. euryale (Nixon, 1974)

626) C. flavipes Cameron, 1891

627) C. geryonis (Marshall, 1885)

628) C. glomerata (Linnaeus, 1758)

629) C. gonopterygis (Marshall, 1897)*

630) C. hyphantriae (Riley, 1887)

631) C. jucunda (Marshall, 1885)

632) C. kazak (Telenga, 1949)

633) C. melanoscela (Ratzeburg, 1844)

634) C. notha (Marshall, 1885)

635) C. ordinaria (Ratzeburg, 1844)

636) C. perspicua (Nees, 1834)

637) C. plutellae (Kurdjumov, 1912)

638) C. praepotens (Haliday, 1834)

639) C. risilis (Nixon, 1974)

640) C. rubecula (Marshall, 1885)

641) C. ruficrus (Haliday, 1834)

642) C. salebrosa (Marshall, 1885)

643) C. saltator (Thunberg, 1822)

644) C. scabricula (Reinhard, 1880)

645) C. sessilis (Geoffroy, 1785)

646) C. setebis (Nixon, 1974)

647) C. specularis (Szépligeti, 1896)

648) C. spuria (Wesmael, 1837)

649) C. telengai (Tobias, 1972)

650) C. tenebrosa (Wesmael, 1837)

651) C. tibialis (Curtis, 1830)

652) C. vanessae (Reinhard, 1880)

653) C. vestalis (Haliday, 1834)

654) C. villana (Reinhard,1880)

655) C. zygaenarum (Marshall, 1885)

656) Deuterixys rimulosa (Niezabitowski, 1910)

657) Diolcogaster alvearia (Fabricius, 1798)

658) D. claritibia (Papp, 1959)

659) D. mayae (Shestakov, 1932)

660) D. spreta (Marshall, 1885)

661) Glyptapanteles porthetriae (Muesebeck, 1928)

662) G. vitripennis (Curtis, 1830)

663) Microgaster australis Thomson, 1895

664) M. globata (Linnaeus, 1758)

665) M. luctuosa Haliday, 1834

666) M. parvistriga Thomson, 1895

667) M. rufipes Nees, 1834

668) Microplitis aduncus (Ruthe, 1860)

669) M. albipennis Abdinbekova, 1969*

670) M. decipiens Prell, 1925

671) M. deprimator (Fabricius, 1798)
672) M. fulvicornis (Wesmael, 1837)

673) M. mandibularis (Thomson, 1895)*

674) M. marshallii Kokujev, 1898

675) M. ochraceus Szépligeti, 1896

676) M. rufiventris Kokujev, 1914

677) M. scrophulariae Szépligeti, 1898

678) M. spectabilis (Haliday, 1834)

679) M. spinolae (Nees, 1834)

680) M. tuberculifer (Wesmael, 1837)

681) M. viduus (Ruthe, 1860)

682) Protapanteles acasta (Nixon, 1973)*

683) P. immunis (Haliday, 1834)

684) P. liparidis (Bouché, 1834)

685) P. mygdonia (Nixon, 1973)

686) P. thompsoni (Lyle, 1927)

\section{Subfamily Microtypinae}

687) Microtypus algiricus Szépligeti, 1908

688) M. desertorum Shestakov, 1932

689) M. trigonus (Nees, 1843) - (in Beyarslan et al., 2017)

690) M. wesmaelii Ratzeburg, 1848

Subfamily Miracinae

691) Centistidea pistaciella van Achterberg \& Mehrnejad, 2002

692) Mirax caspiana Farahani, Talebi, van Achterberg \& Rakhshani, 2014

693) M. rufilabris Haliday, 1833

\section{Subfamily Opiinae}

694) Apodesmia damnosa (Papp, 1980)

695) A. irregularis (Wesmael, 1835)

696) A. karesuandensis (Fischer, 1964)

697) A. novosimilis (Fischer, 1989)

698) A. nowakowskii (Fischer, 1959)

699) A. rex (Fischer, 1958)

700) A. sharynensis (Fischer, 2001)

701) A. similis (Szépligeti, 1898)

702) A. tirolensis (Fischer, 1958)

703) A. tuberculata (Fischer, 1959)

704) A. uttoisimilis (Fischer, 1999)

705) Atormus victus (Haliday, 1837)

706) Biosteres arenarius (Stelfox,1959)

707) B. blandus (Haliday, 1837)

708) B. brevipalpis (Thomson, 1895)

709) B. carbonarius (Nees, 1834)

710) B. haemorrhoeus (Haliday, 1837)

711) B. longicauda (Thomson, 1895)

712) B. punctiscuta (Thomson, 1895)

713) B. remigii Fischer, 1971

714) B. rusticus (Haliday, 1837)

715) Biosteres scabriculus (Wesmael, 1835) (in Ghahari \& Beyarslan, 2017)

716) B. spinaciae (Thomson, 1895)

717) B. ultor (Förster, 1863)

718) B. wesmaelii (Haliday, 1837) 
719) Eurytenes abnormis (Wesmael, 1835)

720) Fopius carpomyiae (Silvestri, 1916)

721) Indiopius cretensis Fischer, 1983

722) Opiognathus propodealis (Fischer, 1958)

723) Opiostomus campanariae (Fischer, 1959)

724) O. riphaeus (Tobias, 1986)

725) O. snoflaki (Fischer, 1959)

726) Opius abditus Fischer, 1960

727) O. aethiops Haliday, 1837

728) O. ambiguus Wesmael, 1835

729) O. arundinis Fischer, 1964

730) O. bajariae Fischer, 1989

731) O. basalis Fischer, 1958

732) O. biroi Fischer, 1960

733) O. biroicus Fischer \& Beyarslan, 2005

734) O. bouceki Fischer, 1958

735) O. caricivorae Fischer, 1964

736) O. caucasi Tobias, 1986

737) O. cingulatus Wesmael, 1835

738) O. circulator (Nees, 1834)

739) O. connivens Thomson, 1895

740) O. coracinus Thomson, 1895

741) O. crassipes Wesmael, 1835

742) O. curticornis Fischer, 1960

743) O. depeculator (Förster, 1863)

744) O. diversiformis Fischer, 1960

745) O. diversus Szépligeti, 1898

746) O. exiguus Wesmael, 1835

747) O. ferrugator (Goureau, 1862)

748) O. flavipes Szépligeti, 1898

749) O. fuscipennis Wesmael, $1835^{*}$

750) O. gracilis Fischer, 1957*

751) O. latidens Fischer, 1990

752) O. latipediformis Fischer, 2004

753) O. latistigma Fischer, 1960

754) O. levis Wesmael, 1835

755) O. longicornis Thomson, 1895

756) O. lonicerae Fischer, 1958

757) O. lugens Haliday, 1837

758) O. magnicauda Fischer, 1958

759) O. minusculae Fischer, 1967

760) O. mirabilis Fischer, 1958

761) O. mirabundus Papp, 1982

762) O. monilicornis Fischer, 1962

763) O. nigricoloratus Fischer, 1958

764) O. nitidulator (Nees, 1834)

765) O. occulisus Telenga, 1950

766) O. opacus Fischer, 1968

767) O. pallipes Wesmael, 1835

768) O. paraplasticus Fischer, 1972

769) O. pendulus Haliday, 1837

770) O. penetrator Fischer, 1966

771) O. ponticus Fischer, 1958

772) O. pulchriceps Szépligeti, 1898

773) O. pumilio Wesmael, 1835

774) O. pygmaeator (Nees, 1811)

775) O. pygmaeus Fischer, 1962
776) O. robustus Telenga, 1950

777) rotundiventris (Thomson, 1895)*

778) O. rudis Wesmael, 1835

779) O. seductus Fischer, 1959

780) O. singularis Wesmael, 1835

781) O. speciosus Fischer, 1959

782) O. staryi Fischer, 1958

783) O. tabificus Papp, 1979

784) O. teheranensis Fischer, 1990

785) O. tersus (Förster, 1863)

786) O. testaceus Wesmael, 1838

787) O. truncatus Wesmael, 1835

788) O. turcicus Fischer, 1960

789) Pokomandya curticornis Fischer, 1959

790) Psyttalia concolor (Szépligeti, 1910)

791) Psyttoma wachsmanni (Szépligeti, 1898)

792) Rhogadopsis reconditor (Wesmael, 1835)

793) Xynobius aciculatus (Thomson, 1895)

794) X. decoratus (Stelfox, 1949)

795) X. macrocerus (Thomson, 1895)

796) X. maculipes (Wesmael, 1835)

797) X. scutellatus (Fischer, 1962)

\section{Subfamily Orgilinae}

798) Kerorgilus zonator (Szépligeti, 1896)

799) Orgilus abbreviator (Ratzeburg, 1852)

800) O. hungaricus Szépligeti, 1896

801) O. ischnus Marshall, 1898

802) O. jennieae Marsh, 1979

803) O. meyeri Telenga, 1933

804) O. nitidior Taeger, 1989

805) O. obscurator (Nees, 1812)

806) O. pimpinellae Niezabitowski, 1910

807) O. ponticus Tobias, 1986

808) O. priesneri Fischer, 1958

809) O. punctiventris Tobias, 1976

810) O. punctulator (Nees, 1812)

811) O. temporalis Tobias, 1976

812) O. tobiasi Taeger, 1989

\section{Subfamily Pambolinae}

813) Pambolus biglumis (Haliday, 1836)

814) Phaenodus pallipes Förster, 1863

\section{Subfamily Rhysipolinae}

815) Cerophanes kerzhneri Tobias, 1971

816) Rhysipolis decorator (Haliday, 1836)

817) Rh. hariolator (Haliday, 1836)

818) Rh. meditator (Haliday, 1836)

Subfamily Rhyssalinae

819) Acrisis brevicornis Hellén, 1957

820) Dolopsidea indagator (Haliday, 1836)

821) Oncophanes minutus (Wesmael, 1838)

Subfamily Rogadinae

822) Aleiodes aestuosus (Reinhard, 1863) 
823) A. agilis (Telenga,1941)

824) A. albitibia (Herrich-Schäffer, 1838) -

(in Beyarslan et al., 2017)

825) A. alternator (Nees, 1834)

826) A. apicalis (Brullé, 1832)

827) A. arnoldii (Tobias, 1976)

828) A. bicolor (Spinola, 1808)

829) A. circumscriptus (Nees, 1834)

830) A. compressor (Herrich-Schäffer, 1838)

831) A. coxalis (Spinola, 1808) -

(in Beyarslan et al., 2017)

832) A. crassipes (Thomson, 1892)

833) A. dimidiatus (Spinola, 1808)

834) A. ductor (Thunberg, 1822)

835) A. esenbeckii (Hartig, 1838)

836) A. eurinus (Telenga, 1941)

837) A. gasterator (Jurine, 1807)

838) A. gastritor (Thunberg, 1822)

839) A. nigricornis Wesmael, 1838

840) A. nocturnus (Telenga, 1941)

841) A. pallescens Hellén, 1927

842) A. pallidator (Thunberg, 1822)

843) Aleiodes pallidicornis (Herrich-Schäffer, 1838)

- (in Ghahari \& Beyarslan, 2017)

844) A. ruficornis (Herrich-Schäffer, 1838) -

(in Beyarslan et al., 2017)

845) A. rufipes (Thomson, 1892)

846) A. seriatus (Herrich-Schäffer, 1838)

847)A. signatus (Nees, 1811)

848) A. testaceus (Spinola, 1808)

849) A. testaceus (Telenga, 1941)

850) A. unipunctator (Thunberg, 1822)

851) A. varius (Herrich-Schäffer, 1838)

852) Clinocentrus cunctator (Haliday, 1836)

853) C. excubitor (Haliday, 1836)

854) C. exsertor (Nees, 1811)

855) C. vestigator (Haliday, 1836)

856) Heterogamus dispar (Haliday, 1833)

887) H. testaceus Telenga, 1941 -

(in Beyarslan et al., 2017)

858) Yelicones iranus (Fischer, 1963)

\section{Subfamily Sigalphinae}

859) Acampsis alternipes (Nees, 1816)

860) Sigalphus caudatus Nees, 1816*

861) S. irrorator (Fabricius, 1775) 